\title{
Influence of purified attapulgite clays on the adhesive properties of cement pastes as measured by the tack test
}

\author{
Shiho Kawashima ${ }^{\mathrm{a}, \mathrm{b}, *}$, Mohend Chaouche ${ }^{\mathrm{c}}$, David J. Corr ${ }^{\mathrm{b}}$, Surendra P. Shah ${ }^{\mathrm{b}}$ \\ ${ }^{a}$ Columbia University, Civil Engineering and Engineering Mechanics, 500 W. 120th Street, 616 SW Mudd, New York, NY 10027, USA \\ ${ }^{\mathrm{b}}$ Northwestern University, Civil and Environmental Engineering, 2145 Sheridan Road, Evanston, IL 60208, USA \\ ${ }^{\mathrm{c}}$ CNRS, Ecole Normale Supérieure de Cachan, Laboratoire de Mécanique et Technologie, 61 Avenue du Président Wilson, 9423 Cachan, France
}

\section{A R T I C L E I N F O}

\section{Article history:}

Received 16 June 2013

Received in revised form 6 January 2014

Accepted 9 January 2014

Available online 24 January 2014

\section{Keywords:}

Attapulgite

Nanoclay

Cement

Rheology

Adhesive properties

Tack test

\begin{abstract}
A B S T R A C T
This study evaluates the influence of small additions of highly-purified attapulgite clays $(0.2 \%$ and $0.5 \%$ addition by mass of cement) on the adhesive properties of cement pastes. Adhesive properties are measured by the tack test, a novel method of evaluating the rheological properties of granular materials. To better understand the results of the tack test as they pertain to cementitious materials, a highly concentrated material that is evolving due to thixotropic rebuilding and hydration, they are supplemented with a measure of the viscoelastic properties over time obtained through low-amplitude oscillatory shear rheometry. The influence of different preshear conditions and resting times (age of paste) on the adhesive properties are determined. Results show the tack test to be a suitable method for obtaining useful information about the adhesive properties and structural evolution of the material in the fresh state.
\end{abstract}

(c) 2014 Elsevier Ltd. All rights reserved.

\section{Introduction}

The fresh-state properties of cementitious materials impact construction productivity and the eventual properties of the material in service. With the addition of chemical and mineral admixtures, it is possible to manipulate the fresh state to improve concrete processing. This has led to such innovations as self-consolidating concrete (SCC) - a highly flowable concrete with superior segregation resistance. These properties effectively eliminate the need to apply external vibration during casting, which increases construction efficiency. However, the high flowability and faster casting rates achieved with SCC compared to normal vibrated concrete results in potentially higher lateral pressure exerted on formwork. Due to this effect, and the lack of a full understanding of the pressure transmitted to formwork from SCC, formwork is typically designed to withstand the full hydrostatic pressure that the SCC could exert. As formwork is one of the largest components of the cost of concrete construction, it is desirable to safely reduce the formwork design criteria through suitable materials selection and proper construction techniques.

The pressure exerted by SCC on formwork is closely tied to its fresh-state rheological properties. Recent work has shown the

* Corresponding author at: 500 W. 120th Street, 616 SW Mudd, Mail Code 4709, New York, NY 10027, USA. Tel.: +1 2128542701 (O).

E-mail address: s-kawashima@columbia.edu (S. Kawashima). potential of further manipulating the rheological properties of SCC with clays. Small additions of clays ( $<1 \%$ by mass of binder) have been found to significantly increase the green strength (fresh-state stiffness) and shape stability of SCC with little compromise to the initial flowability [1]. The most pronounced effect was achieved with nanoclays (clays dispersed into their individual constituents). These properties were demonstrated to be effective in reducing SCC formwork pressure. In a study that evaluated the influence of different mineral admixtures on the formwork pressure response of SCC, an SCC mix with $0.33 \%$ nanoclay was found to experience reduced lateral pressure while still exhibiting comparable initial slump flow as the control [2].

The physical origin of this effect of clays is not fully understood. Flocculation studies have shown that clays increase floc size and flocculation strength $[3,4]$, which can increase the viscosity and level of thixotropy of concrete. Reduction in SCC formwork pressure has been tied to increases in thixotropy [5-7]. In a series of studies determining the influence of various admixtures on SCC formwork pressure, thixotropy-enhancing admixtures were found to decrease initial pressure and increase rate of pressure drop over time [8-10]. Although thixotropy is undoubtedly tied to the clay effect, the cohesiveness within the material is expected to have a significant effect on stress transfer and subsequently on the formwork pressure. This issue can be investigated by considering the adhesive properties of the material. A standard method of evaluating the adhesive properties of a material is the probe tack test, which is widely used to 
complement rheological characterization of soft polymer adhesives [11-13]. The test can provide a measure of cohesion (the internal strength of the material at rest) and adherence (the tendency of the material to stick to a surface). High cohesion has been found to increase the rate of lateral pressure drop of SCC over time [14]. And high adherence can help to transfer lateral stress to vertical stress, making it possible for SCC formwork pressure to be less than hydrostatic [15]. Therefore, the influence of clays on the adhesive properties of cementitious materials is of interest.

Thus far, only a few select studies have implemented the tack test on granular-type materials $[16,17]$. Due to the limited number of studies that have been done to evaluate the adhesive properties of cementitious materials, the information that can be obtained from the results of the tack test is not fully resolved. In addition, due to the nature of the material: a highly concentrated suspension that is evolving due to thixotropic rebuilding and hydration, there is a need to interpret the results while taking the aging of the material into careful consideration. This current study aims to evaluate the influence of nanoclays on the adhesive properties of cement pastes through the tack test. In addition, to better understand the results as they pertain to cementitious materials in general, they are supplemented with a measure of the viscoelastic properties over time through lowamplitude oscillatory shear rheometry. It is found that the tack test is a suitable method for obtaining useful information about the adhesive properties and structural evolution of the material in the fresh state.

\section{Materials and experimental procedures}

\subsection{Materials}

Tap water and type I Portland cement with a Blaine fineness of $385 \mathrm{~m}^{2} / \mathrm{kg}$ are used in all mixes. A highly-purified attapulgite clay, refined from bulk attapulgite, is the clay selected for this study [18]. They are $1.75 \mu \mathrm{m}$ in average length and $3 \mathrm{~nm}$ in average diameter, thereby considered to be nano-sized. They have been chemically exfoliated to preserve their uniform shape and size while removing all impurities (such as quartz and swelling clays). The aspect ratio (average length divided by average diameter $=583$ ) of the clay particles is very high. Therefore, they may form a highly entangled gel even at a small volume concentration, provided they are properly dispersed into individual particles.

All paste mixes have a water-to-cement $(\mathrm{w} / \mathrm{c})$ ratio of 0.43 by mass and are prepared by hand-stirring for $60 \mathrm{~s}$. For mixes with a nanoclay addition, they are introduced as an aqueous suspension, where the nanoclay is blended with the mixing water in a household blender for 5 min to facilitate dispersion. All tests start immediately after mixing.

\subsection{Experimental methods}

All rheological tests are performed on a Paar Physica MCR rheometer with a parallel-plate geometry. The top plate has a diameter of $50 \mathrm{~mm}$ and the bottom plate is temperature-controlled with a circulating water bath set to $20^{\circ} \mathrm{C}$. To prevent slip, the surfaces of the plates are covered with 150-grit adhesive sandpaper. The rate of data acquisition is $10 \mathrm{~Hz}$ for higher plate velocities and $5 \mathrm{~Hz}$ for lower plate velocities. Further details of the experimental method can be found elsewhere [19].

\subsubsection{Tack test}

The tack test is performed on cement pastes with $0 \%, 0.2 \%$ and $0.5 \%$ nanoclay addition by mass of cement ( $0 \mathrm{NC}, 0.2 \mathrm{NC}$ and 0.5
NC). The fresh cement paste sample is placed on the bottom plate, then the top plate is lowered to an initial measuring gap of $1 \mathrm{~mm}$. Once the top plate is in position, the sample is trimmed to match the diameter of the plate. During the test, the top plate moves up vertically at a constant velocity, subjecting the sample to stretching, and the normal force experienced by the top plate is recorded over time. The normal force evolution is recorded for the following plate velocities: $10,50,200,500$, and $1000 \mu \mathrm{m} / \mathrm{s}$. The test is run until the sample reaches complete separation between the two plates. A shear rate of $50 \mathrm{~s}^{-1}$ (measured at the circumference of the sample) is applied for $60 \mathrm{~s}$ before the start of the tack test to ensure all samples start at the same state after mixing.

Cementitious materials are both time and shear history dependent. Therefore, the influence of a higher preshear condition and various resting times on the adhesive response of pastes is evaluated. The tack test is performed at $10 \mu \mathrm{m} / \mathrm{s}$ for cement paste mixes with a $0 \%$ and $0.5 \%$ nanoclay addition subjected $300 \mathrm{~s}^{-1}$ for $60 \mathrm{~s}$. Following this preshear, the tack test is performed after the following resting periods: 0,150 , and $300 \mathrm{~s}$.

A new sample is prepared for each test. Three measurements are taken for each mix for each testing protocol and the average is taken to be the representative measurement.

\subsubsection{Low-amplitude oscillatory shear rheometry}

To better interpret the results of the tack test, they are supplemented with a measure of the evolution of the paste's structure. This is done through low-amplitude oscillatory shear rheometry. This method provides information about the viscoelastic properties of suspensions and it has been demonstrated to be applicable to fresh cement paste $[20,21]$. The theory is briefly explained here.

An oscillatory strain is applied as a sine function:

$\gamma=\gamma_{0} \sin \omega t$

where $\gamma_{0}$ is maximum strain amplitude, $t$ is time, and $\omega$ is frequency. If the strain is sufficiently low so that the particles in the suspension remain close to each other, the microstructure is not disturbed and the material can recover elastically. In this case (linear regime), the measured response in terms of stress is as follows:

$\tau=\gamma_{0}\left(G^{\prime} \sin \omega t+G^{\prime \prime} \cos \omega t\right)$

where $\tau$ is shear stress, $G^{\prime}$ is storage modulus, and $G^{\prime \prime}$ is loss modulus. $G^{\prime}$ is the elastic (or in-phase) component while $G^{\prime \prime}$ is the viscous (or out-of-phase) component. By monitoring the time evolution of $G^{\prime}$, it is possible to measure the structural building of cement pastes over time. The method has been applied to determine the effect of admixtures, such as nanosilica and cellulose ethers, and w/c ratio on the storage modulus of cement pastes and has been tied to flocculation and hydration mechanisms [22-24].

The first step in performing oscillatory shear rheometry is to find the linear viscoelastic region (LVR), where $G^{\prime}$ (and also $G^{\prime \prime}$ ) is independent of applied frequency and strain. To find the LVR, a strain sweep is performed on cement pastes with $0 \%$ and $0.5 \%$ nanoclay addition. Strain amplitudes ranging from $10^{-4}$ to 1 are applied at a fixed frequency of $1 \mathrm{~Hz}$. The amplitude sweep results are reported in Fig. 1a. The strain sweep curves exhibit two main branches - a plateau region corresponding to the LVR regime and a decreasing branch indicating that the sample is experiencing a shear-induced breakage. The LVR regime of the plain cement paste ends at a critical strain of about $6 \times 10^{-4}$, which corresponds to the same order of magnitude as that reported by previous authors [21]. The critical strain for the clay-modified cement paste is significantly higher than that of a plain cement paste. This is in agreement with previously reported results indicating that the addition of clays increases flocculation within the 

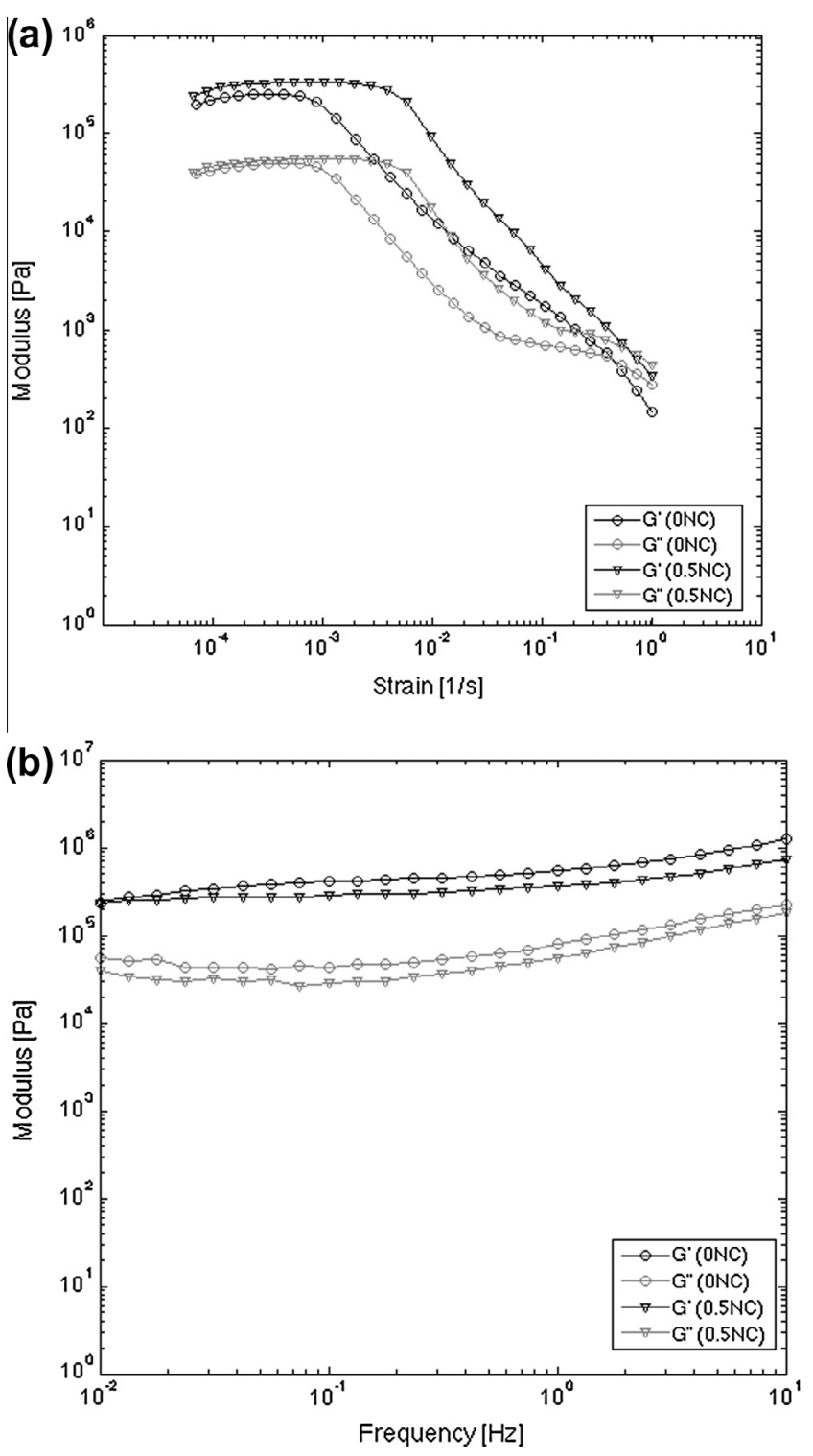

Fig. 1. (a) Strain and (b) frequency sweeps of cement pastes with $0 \%$ and $0.5 \%$ nanoclay addition [19].

paste $[3,4]$. A similar effect on critical strain was shown with cellulose ethers, which the researchers attributed to adsorption of the polymer on cement particles and subsequent strengthening of the gel structure [23].

A frequency sweep experiment is performed in which frequencies from $0.01 \mathrm{~Hz}$ to $10 \mathrm{~Hz}$ are applied at a fixed strain of $10^{-3}$. The frequency sweep results are shown in Fig. 1b. For both samples there is no significant decrease of $G^{\prime}$ at low frequencies, indicating that they comprise of a highly gelled structure. Moreover $G^{\prime}$ remains higher than $G^{\prime \prime}$ throughout the frequency range considered.

Based on these results, an applied strain of $10^{-4}$ and frequency of $1 \mathrm{~Hz}$ are selected for the oscillatory shear measurements in order to remain in the linear regime. Oscillatory shear measurements are performed on cement pastes with $0 \%$ and $0.5 \%$ nanoclay addition. The evolution of storage modulus is monitored for $900 \mathrm{~s}$ (approximately the duration of the tack test at the smallest pulling velocity considered, $10 \mu \mathrm{m} / \mathrm{s})$. The oscillatory tests are performed for the same preshear conditions as for the tack tests: $50 \mathrm{~s}^{-1}$ for $60 \mathrm{~s}$ and $300 \mathrm{~s}^{-1}$ for $60 \mathrm{~s}$.

\section{Results and discussion}

\subsection{Normal force evolution at various plate velocities}

The influence of nanoclay on the adhesive properties of cement pastes is evaluated by the tack test, where the normal force evolution is recorded for various plate velocities. At the very beginning of the test, the sample deforms elastically and the normal force increases with the gap thickness (or time). Beyond a critical tensile strain the normal force decreases, indicating that the sample starts undergoing a failure process, including inward flow towards the center of the plate. The first value of interest is the peak force, corresponding to the critical strain. The value of the peak force may comprise of at least two contributions: resistance to flow (viscous effects) and resistance to elastic failure due to the intrinsic cohesion of the material. The physical origin of cohesion may include intermolecular forces and capillary effects [16] (bubbles are always present in the sample). The cohesion strength may be related to the yield stress but under stretching conditions. The strength of the material cohesion may be considered to correspond to the value of the peak force for a vanishingly small pulling velocity. In the present investigation the cohesion component is assumed to be the value of the peak force for the smallest velocity considered, $10 \mu \mathrm{m} / \mathrm{s}$. The results concerning this first part of the normal force behavior are similar to those reported in the literature concerning other types of cementitious materials [17]. However, as it can be seen below, the behavior of the force beyond the peak value is qualitatively different from the previously reported tack test results.

The normal force evolution of cement pastes with $0 \%, 0.2 \%$ and $0.5 \%$ nanoclay addition are compared and the results are shown in Figs. 2-4, respectively. It is apparent that even at small additions, the nanoclay significantly increases the normal force experienced by the cement pastes at all plate velocities. This indicates that the clay increases cohesion and viscous dissipation. To better illustrate this effect, the peak force versus plate velocity is plotted for all mixes. The results are shown in Fig. 5. For each paste the peak force increases globally with plate velocity. This is expected and can be explained by the increase in the viscous dissipation with increasing velocity. Yet, the tack flow curves seem to reach a plateau beyond $200 \mu \mathrm{m} / \mathrm{s}$. Then, the rheological behavior under extension is similar to that under shear and can be interpreted in a

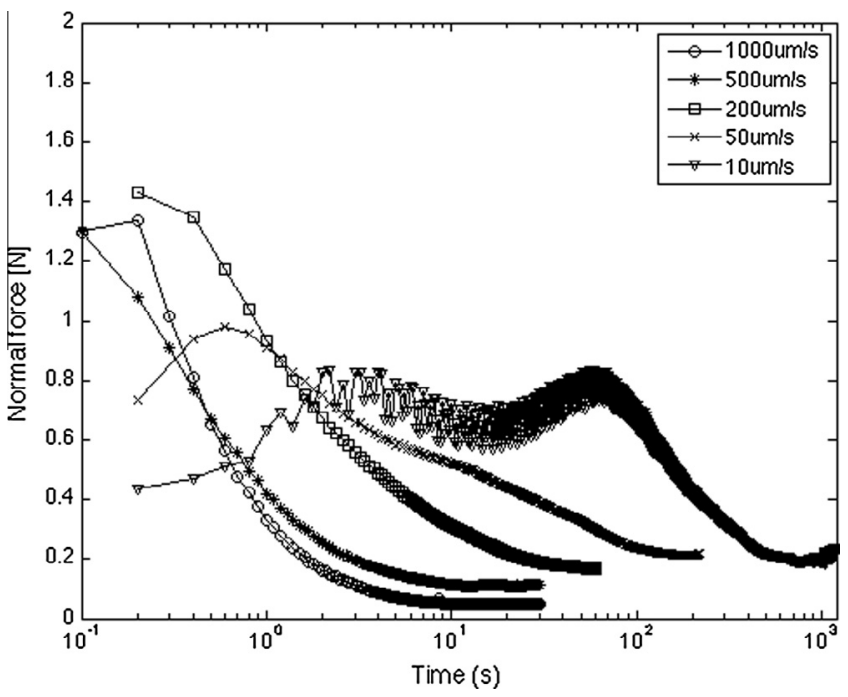

Fig. 2. Normal force evolution curves for plain cement paste [19]. 


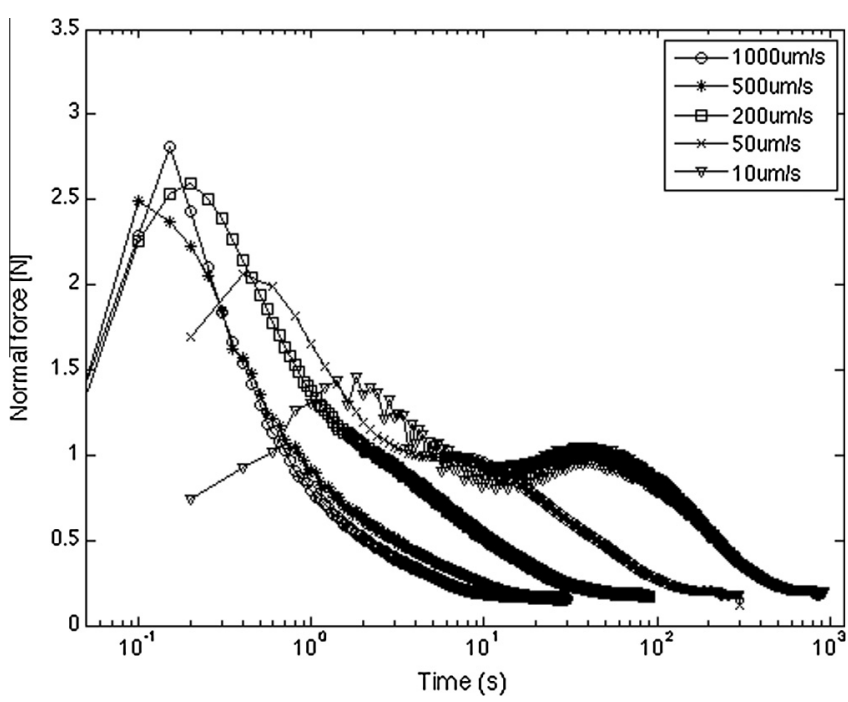

Fig. 3. Normal force evolution curves for cement paste with $0.2 \%$ nanoclay addition [19].

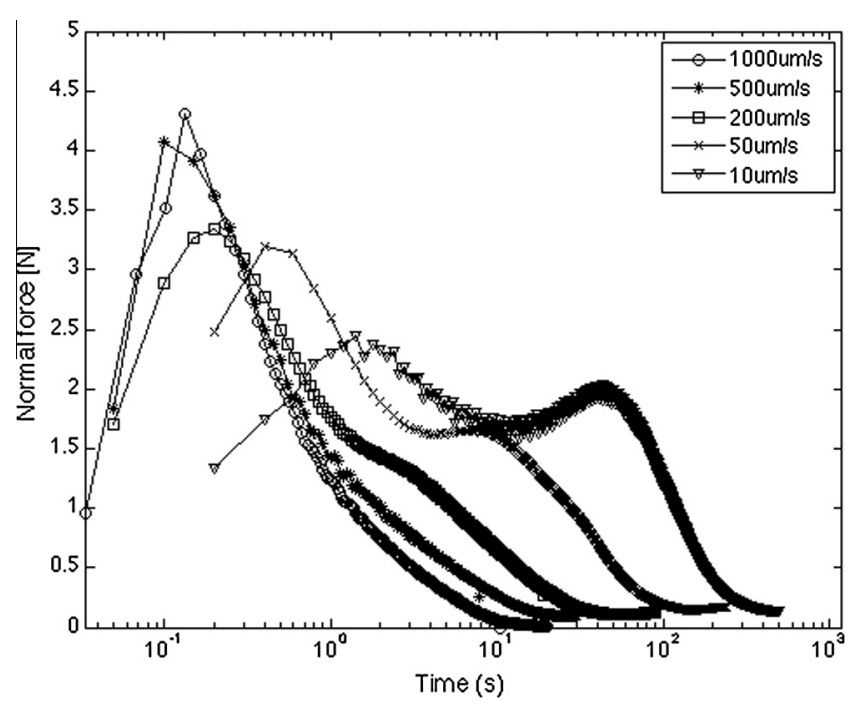

Fig. 4. Normal force evolution curves for cement paste with $0.5 \%$ nanoclay addition [19].

similar manner. Further, the clay-modified pastes experience higher velocity dependence compared to the plain cement paste. This is due to the increase in viscosity caused by the clays, the origin of which can be tied to flocculation. According to the KriegerDougherty equation [25], which describes the flow behavior of highly-concentrated suspensions, a system with a lower maximum packing density (more flocculated) will exhibit higher viscosity [3].

An interesting feature in the normal force evolution for all paste mixes is the occurrence of a second peak (strain hardening). It is the most marked at the lowest plate velocity, $10 \mu \mathrm{m} / \mathrm{s}$, but also evident at 50 and $200 \mu \mathrm{m} / \mathrm{s}$. It suggests that the material is exhibiting residual resistance to the plates separating after the break in flow indicated by the initial peak. Similar strain hardening has been observed in polymer adhesives tested under spherical and flat punches $[13,11]$, where higher initial maximum contact radiusto-height ratios made this effect more pronounced. This strain hardening was attributed to fibrillation and is akin to polymer materials. On the other hand, this effect was not observed in a study where the tack test was performed on muds, even though

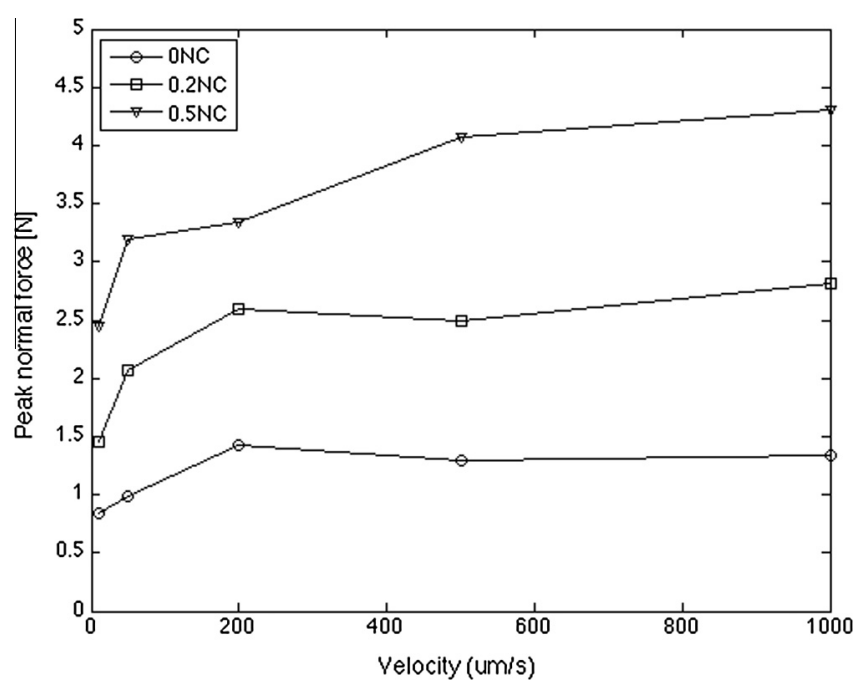

Fig. 5. Peak force vs plate velocity for cement pastes with $0 \%, 0.2 \%$ and $0.5 \%$ nanoclay addition [19].

the initial maximum contact radius-to-height ratio was higher than the one used in the present study [16]. Even in the case of the previously reported results on cement-based materials, strain hardening was not observed [26,17]. This may be attributed to the difference in experimental protocol. In those studies the tack tests were performed at certain waiting times and this has critical effects, as it will be seen later. Given the time-dependent nature of cementitious materials, this secondary peak can be accounted for by the evolution of its structure due to flocculation and hydration mechanisms. In order to elucidate this, low-amplitude oscillatory shear rheometry is implemented and will be discussed in the next section.

\subsection{Influence of preshear condition}

The occurrence of the secondary peak in the normal force evolution in all mixes may be attributed to the eventual evolution of the material during the course of stretching. The fact that the secondary peak becomes more pronounced when decreasing tack velocity may reflect the fact that one has a competition between two kinetics phenomena: (1) failure of sample whose kinetics is controlled by the applied velocity and its rheology and (2) time evolution of the material due to reflocculation and hydration. To determine the kinetics of the second phenomena, time evolution of the storage modulus is monitored for different preshear conditions. Since the second peak is most pronounced for $10 \mu \mathrm{m} / \mathrm{s}$, the duration of the oscillatory test is selected to match that of the tack test at this plate velocity. In addition, the influence of preshear is determined for both tack and oscillatory measurements. (Note: The duration of mixing and preshear is the same between both tests so the ages of the samples correspond.)

The results of the tack test at $10 \mu \mathrm{m} / \mathrm{s}$ for cement pastes subjected to a low $\left(50 \mathrm{~s}^{-1}\right)$ and high $\left(300 \mathrm{~s}^{-1}\right)$ preshear are shown in Figs. 6 and 7 for $0 \%$ and $0.5 \%$ nanoclay addition, respectively. First, the influence of preshear in each system will be discussed. For the plain cement paste, the initial peak is slightly lower for the sample subjected to $300 \mathrm{~s}^{-1}$ than $50 \mathrm{~s}^{-1}$. This can be explained by the higher degree of structural breakdown that results from a higher preshear. At the time the initial peak occurs, it is less cohesive than the paste that is subjected to the lower preshear. In the cement paste with clay, the response is markedly different. Although at the very beginning (up to $0.3 \mathrm{~s}$ ), the paste subjected to $300 \mathrm{~s}^{-1}$ exhibits lower normal force, it quickly recovers and goes onto ex- 


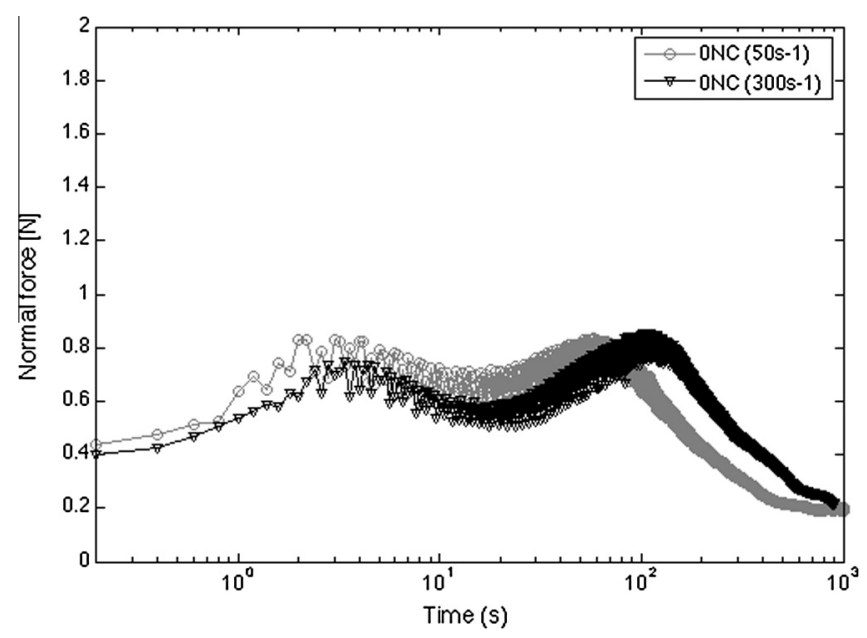

Fig. 6. Influence of preshear on normal force evolution at $10 \mu \mathrm{m} / \mathrm{s}$ for plain cement paste [19].

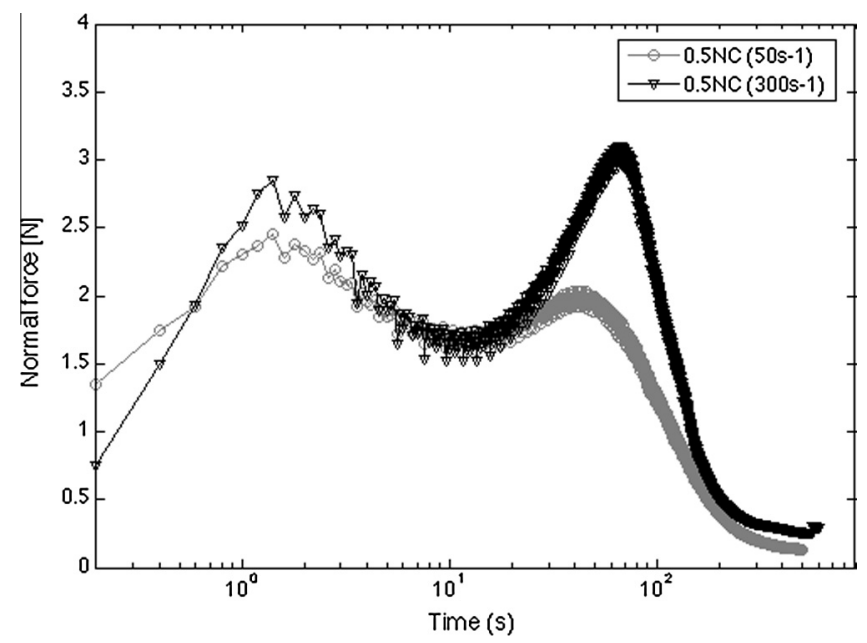

Fig. 7. Influence of preshear on normal force evolution at $10 \mu \mathrm{m} / \mathrm{s}$ for cement paste with $0.5 \%$ nanoclay addition [19].

hibit a higher initial peak than the paste subjected to $50 \mathrm{~s}^{-1}$. This may be attributed to the increase in flocculation kinetics due to the presence of the clay, which results in rapid recovery of the structure. It follows that not only do clays increase the cohesion and viscosity of cement paste (as indicated by the higher normal force experienced over the duration of the test), they accelerate the material's immediate rate of recovery, as well.

For both pastes, there is a second peak that occurs around $100 \mathrm{~s}$. This is likely due to structural thixotropic rebuilding and hydration. The structural evolution of the material at rest experienced after each preshear condition is determined by following the time evolution of $G^{\prime}$ which is plotted for the plain and clay-modified cement pastes in Figs. 8 and 9, respectively. It is apparent that both pastes experience significant structural recovery starting at approximately $10 \mathrm{~s}$. The kinetics of the $G^{\prime}$ evolution can be split into three parts. During the first minute the evolution takes place at a relatively low rate. At around $100 \mathrm{~s}$, we observe a net acceleration of structural evolution. Beyond $10 \mathrm{~min}$, the rate of material evolution starts to decrease, eventually reaching a plateau that can be clearly seen in the case of the clay-modified paste subjected to high preshear. By comparing the tack (Figs. 6 and 7) and oscillatory results (Figs. 8 and 9), it can be seen that the beginning of strain hardening is clearly correlated with the acceleration of the

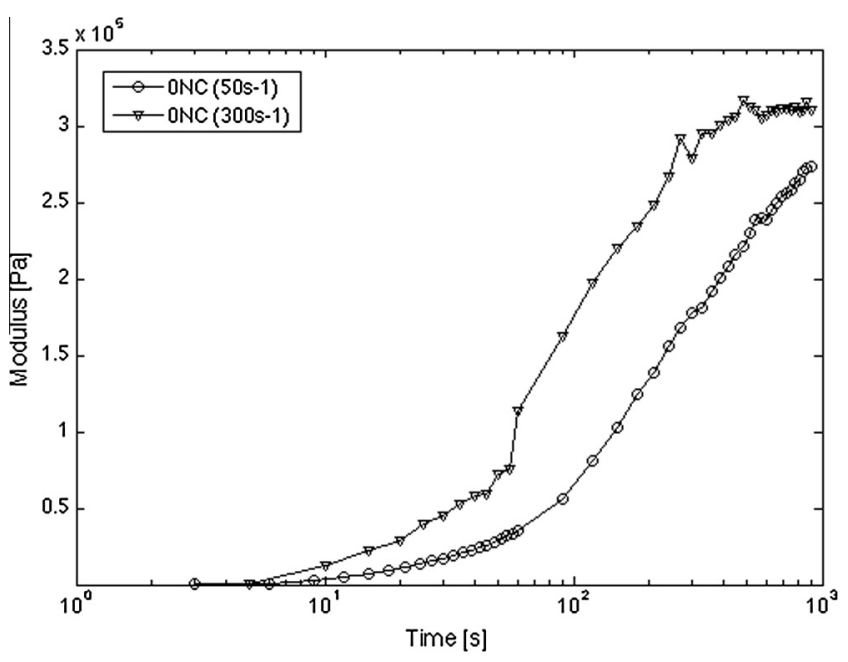

Fig. 8. Evolution of storage modulus of plain cement paste subjected to different preshear conditions [19].

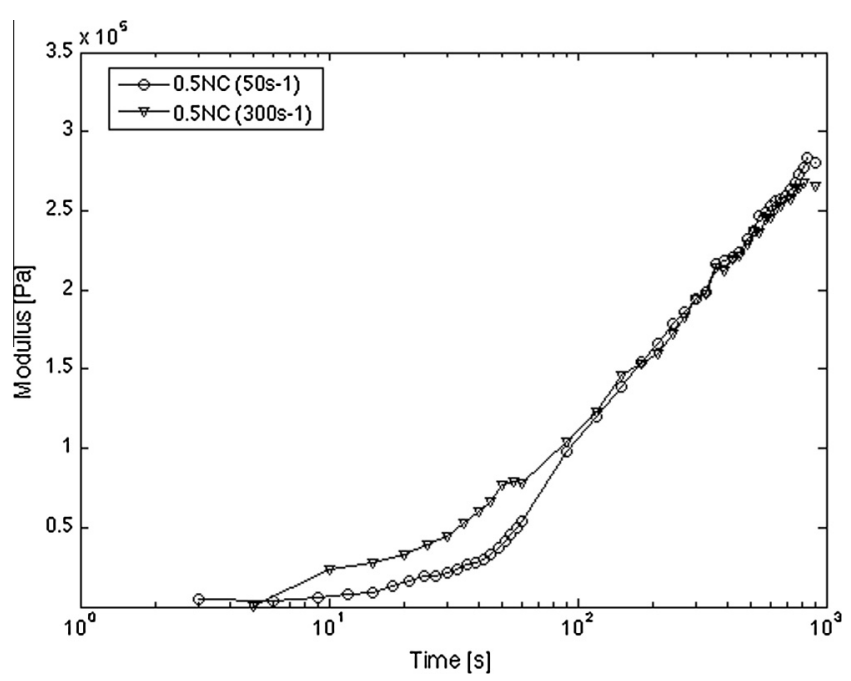

Fig. 9. Evolution of storage modulus of cement paste with $0.5 \%$ nanoclay addition subjected to different preshear conditions [19].

structural evolution of the material. This is likely tied to both physical reflocculation and hydration mechanisms. Studies have shown that as soon as cement and water make contact, a gel of various hydrates forms within the pore solution and on the cement particle [27-29]. The alumina phases are the first to react leading to the formation of ettringite (AFt) (by reaction with gypsum). These needle-like particles may easily give rise to a gel-like structure, leading to an increase of $G^{\prime}$. Other phases may also form at the very beginning of the hydration process (e.g. AFm, portlandite, and early CSH) and may contribute to $G^{\prime}$. The origin of the structural evolution of the pastes may also include the change in ionic concentration or $\mathrm{pH}$ of the pore solution, which occurs very rapidly within the first $20 \mathrm{~min}$. This will lead to a change in the colloidal interactions, subsequently leading to flocculation under certain conditions. For example clay particles are known to flocculate at high ionic strength [30].

In both cases, the storage modulus is higher for the paste subjected to a $300 \mathrm{~s}^{-1}$ preshear - in the plain cement paste it is higher throughout and for the clay-modified paste it is higher from 10 to $100 \mathrm{~s}$. This agrees well with the tack results, where the second peak is higher for the $300 \mathrm{~s}^{-1}$ preshear than for $50 \mathrm{~s}^{-1}$ in both systems with and without clay. This indicates that both pastes experience 
shear thickening. This may be attributed to fact that rebuilding kinetics due to Brownian motion is higher for smaller aggregates, which are obtained at high preshear.

After the second peak, the normal force goes to zero while the storage modulus continues to increase. This is because by the time the second peak is reached, the plates have separated to a point where the area of contact between the sample and the plate surfaces has substantially decreased or the sample has experienced fibrillation. Therefore, the tack test can no longer capture the structural recovery experienced by the paste.

In comparing the post-peak behavior of the pastes, they suggest that the mechanism behind the failure is different in each case. The plain cement paste experiences a more ductile failure while the paste with clay fails more abruptly, with a steeper downward slope. This suggests that the plain cement paste fails in a more liquid-like manner while the clay-modified paste fails in a more solid-like manner. This is captured in the images of the tested samples, shown in Figs. 10 and 11. Upon visual observation, it is apparent that the plain cement paste undergoes a gradual inward flow while the paste with clay is stiffer and undergoes failure within the sample under extension.

\subsection{Influence of rest time}

To determine the influence of resting time on the pastes, the tack test is performed after the sample is allowed to rest for 150 and $300 \mathrm{~s}$ after a $300 \mathrm{~s}^{-1}$ preshear is applied. Through the rest time parameter, it is possible to determine the influence of the age of the sample on its tack properties. The results are shown in Figs. 12 and 13. Both pastes exhibit the same trends. As expected, the normal force increases with resting time. This is due to the structural recovery (and hydration) of the pastes over time, as verified by the evolution of storage modulus presented in the previous section. Also, the second peak is significantly lower than in the previous results, where the tack tests was performed while the sample was evolving rapidly. For the clay-modified paste with a 300 s rest time, it is no longer a peak but a slight hump. This is due to the decrease in the rate of rebuilding at the later ages of the pastes, as it can be seen in the $G^{\prime}$ results. According to the results of oscillatory shear rheometry, $G^{\prime}$ exhibits a more substantial increase from 10 to $100 \mathrm{~s}$ (about $150 \mathrm{kPa}$ for $0 \mathrm{NC}, 80 \mathrm{kPa}$ for $0.5 \mathrm{NC}$ ) than 150 to $250 \mathrm{~s}$ (about $50 \mathrm{kPa}$ for $0 \mathrm{NC}, 30 \mathrm{kPa}$ for $0.5 \mathrm{NC}$ ). The rate of increase in $G^{\prime}$ goes down further after $300 \mathrm{~s}$, tending to a plateau.

These results confirm the fact that the secondary peak that appears in the tack force curves is due to the structural evolution of the material. In addition, all tests are performed with a top plate

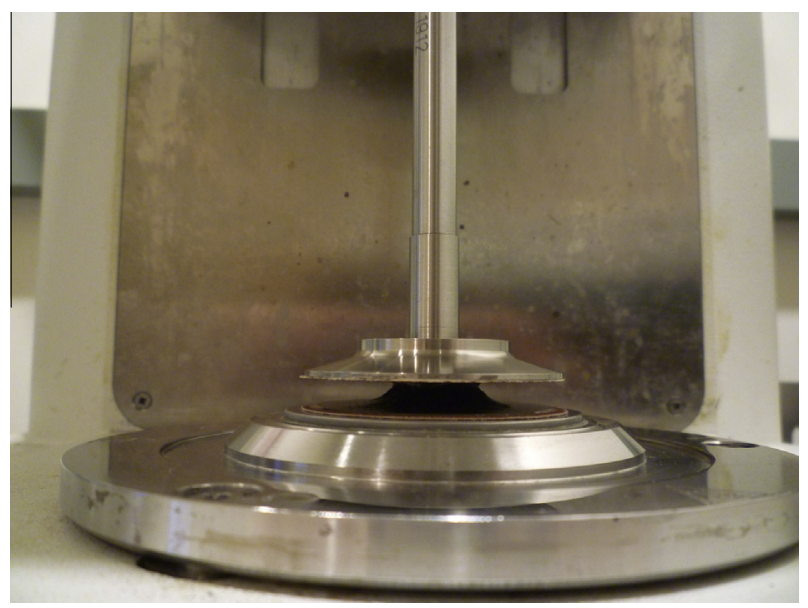

Fig. 10. Plain cement paste during tack test at $10 \mu \mathrm{m} / \mathrm{s}[19]$.

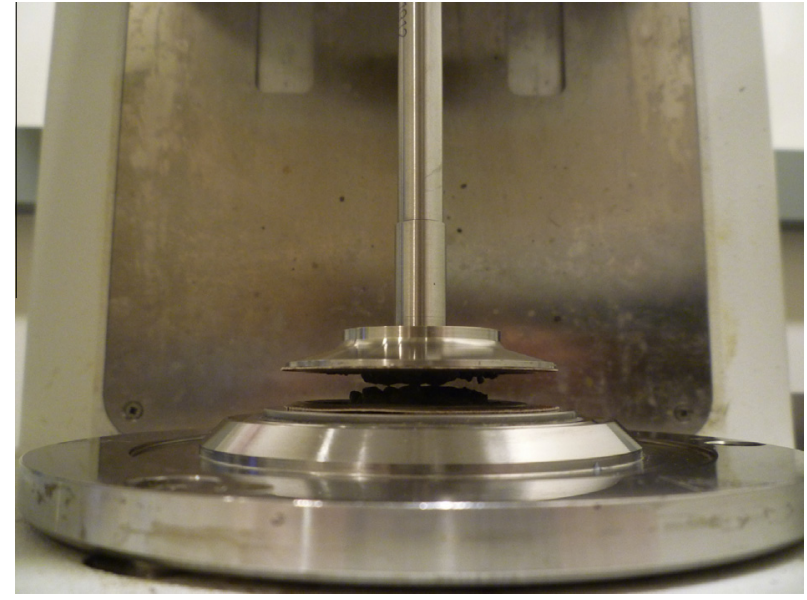

Fig. 11. Cement paste with $0.5 \%$ nanoclay addition during tack test at $10 \mu \mathrm{m} / \mathrm{s}$ [19].

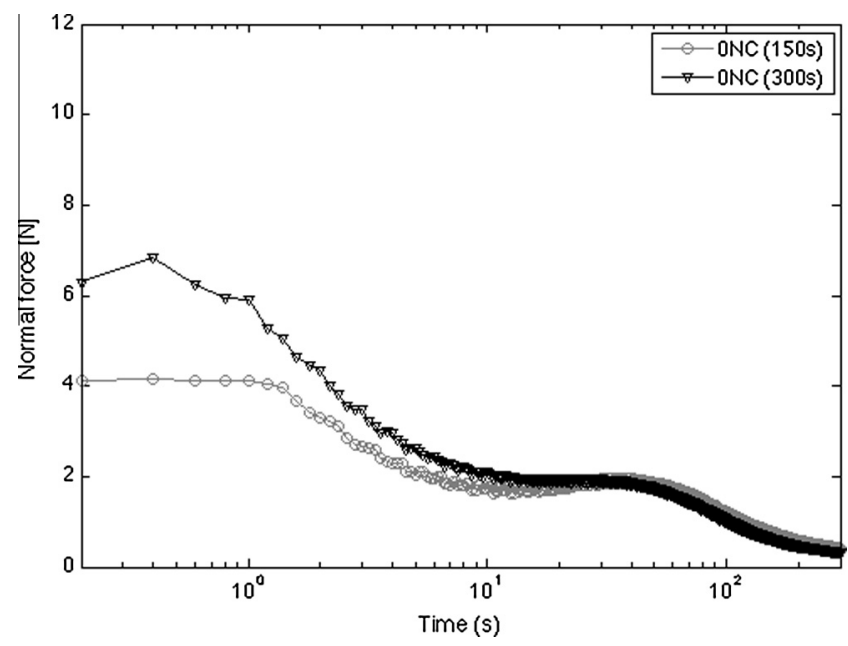

Fig. 12. Influence of resting time on plain cement paste subjected to a $300 \mathrm{~s}^{-1}$ preshear condition [19].

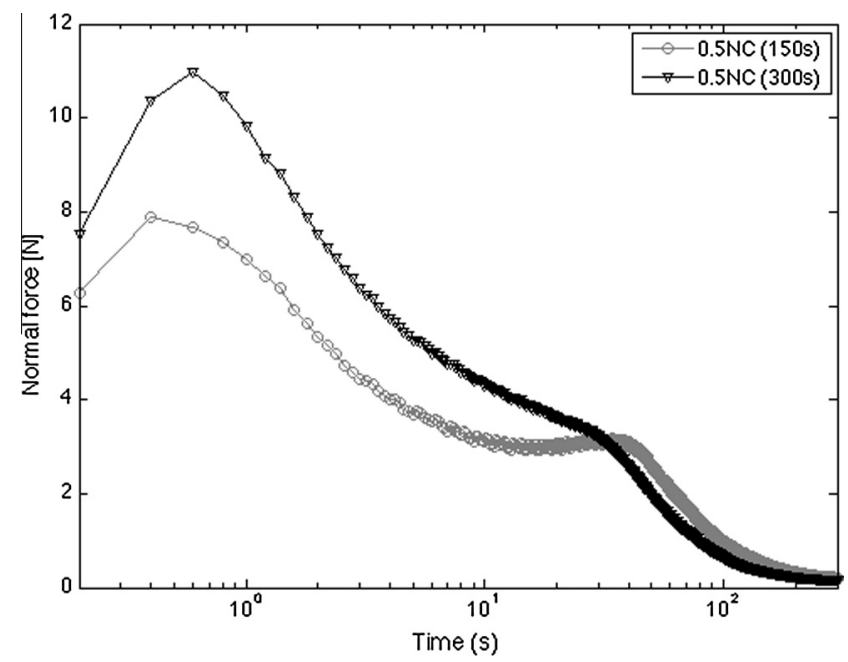

Fig. 13. Influence of resting time on cement paste with $0.5 \%$ nanoclay addition subjected to a $300 \mathrm{~s}^{-1}$ preshear condition [19].

diameter and sample diameter of $50 \mathrm{~mm}$. Size effect is expected $[11,13]$, and determining the relationship between sample size 
and adhesive properties as measured by the tack test is a potential direction of future work.

\section{Conclusions}

This study examined the influence of nanoclay on the adhesive properties of cement pastes through the tack test. To better interpret the results, the normal force evolution curves are supplemented with measures of structural rebuilding of the pastes over time by performing oscillatory rheometry experiments. The key results are as follows:

- At additions of $0.2 \%$ and $0.5 \%$, the clay increases the peak normal force experienced by cement pastes at all plate velocities, indicating increase in cohesion and viscosity. The clay also increases the plate velocity dependence, where higher velocity leads to higher peak force.

- Cement pastes with and without clay exhibit a secondary peak in the normal force evolution curves at lower plate velocities. Oscillatory shear rheometry results show that this is due to the high rate of rebuilding of the structure around this age ( $\sim 10 \mathrm{~s}$ from the start of the test). The origin of the rebuilding can be tied to flocculation and hydration mechanisms.

- Cement pastes with and without clay exhibit a higher secondary peak and increased $G^{\prime}$ after being subjected to the higher preshear. Further, the clay-modified paste exhibits a higher initial peak, as well, indicating that the clay increases the immediate rate of rebuilding. This can be tied to increase in flocculation caused by the clay.

- After a certain resting time, the initial peak increases while the second peak becomes less pronounced. The former can be attributed to the higher degree of recovery and cohesion that the paste achieves after rest. The latter can be attributed to the decrease in rate of structural rebuilding at later ages, as was shown in the evolution of storage modulus.

\section{Acknowledgments}

The authors would like to acknowledge the financial support from the Infrastructure Technology Institute at Northwestern University and Tennessee Valley Authority (TVA) and Oak Ridge Associated Universities (ORAU) (Award 105866); and Active Minerals, WR Grace and Lafarge for providing materials.

\section{References}

[1] Pekmezci BY, Voigt T, Kejin W, Shah SP. Low compaction energy concrete for improved slipform casting of concrete pavements. ACI Mater 2007;104(Compendex):251-8.

[2] Kim JH, Beacraft M, Shah SP. Effect of mineral admixtures on formwork pressure of self-consolidating concrete. Cem Concr Compos 2010;32(9):665-71.
[3] Ferron RD, Shah S, Fuente E, Negro C. Aggregation and breakage kinetics of fresh cement paste. Cem. Concr. Res. 2013;50:1-10.

[4] Tregger NA, Pakula ME, Shah SP. Influence of clays on the rheology of cement pastes. Cem Concr Res 2010;40(Compendex):384-91.

[5] Assaad J, Khayat KH, Mesbah H. Variation of formwork pressure with thixotropy of self-consolidating concrete. ACI Mater J 2003;100(1):29-37.

[6] Ovarlez G, Roussel N. A physical model for the prediction of lateral stress exerted by self-compacting concrete on formwork. Mater Struct 2006;39(2):269-79.

[7] Khayat KH, Omran AF. Field validation of SCC formwork pressure prediction models. ACI Mater J 2011;33(6):33-9.

[8] Assaad J, Khayat KH. Effect of viscosity-enhancing admixtures on formwork pressure and thixotropy of self-consolidating concrete. ACI Mater J 2006;103(4):280-7.

[9] Khayat KH, Assaad J. Effect of w/cm and high-range water-reducing admixture on formwork pressure and thixotropy of self-consolidating concrete. ACI Mater J 2006;103(3):186-93.

[10] Khayat KH, Assaad JJ. Use of thixotropy-enhancing agent to reduce formwork pressure exerted by self-consolidating concrete. ACI Mater J 2008;105(1):88-96.

[11] Lakrout H, Creton C, Ahn D, Shull KR. Influence of molecular features on the tackiness of acrylic polymer melts. Macromolecules 2001;34(21):7448-58.

[12] Drzal PL, Shull KR. Adhesive failure of model acrylic pressure sensitive adhesives. J Adhes 2005;81(3):397-415.

[13] Crosby AJ, Shull KR. Adhesive failure analysis of pressure-sensitive adhesives. J Polym Sci Part B: Polym Phys 1999;37(24):3455-72.

[14] Assaad J, Khayat KH. Formwork pressure of self-consolidating concrete made with various binder types and contents. ACI Mater J 2005;102(4):215-23.

[15] Tchamba JC, Amziane S, Ovarlez G, Roussel N. Lateral stress exerted by fresh cement paste on formwork: laboratory experiments. Cem Concr Res 2008;38(4):459-66.

[16] Mohamed Abdelhaye YO, Chaouche M, Van Damme H. The tackiness of smectite muds. 1. The dilute regime. Appl Clay Sci 2008;42(1-2):163-7.

[17] Kaci A, Bouras R, Phan VT, Andréani PA, Chaouche M, Brossas H. Adhesive and rheological properties of fresh fibre-reinforced mortars. Cem Concr Compos 2011;33(2):218-24.

[18] Active Minerals Company LLC. What is Acti-Gel 208 and how is it made?; 2007.

[19] Kawashima S. Nanomodification of cementitious materials: fresh state and early age. PhD dissertation. Northwestern University, Evanston IL: ProQuest/ UMI; 2013. p. 196 [Publication No. AAT 3556618].

[20] Papo A, Caufin B. A study of the hydration process of cement pastes by means of oscillatory rheological techniques. Cem Concr Res 1991;21(6):1111-7.

[21] Schultz MA, Struble LJ. Use of oscillatory shear to study flow behavior of fresh cement paste. Cem Concr Res 1993;23(2):273-82.

[22] Sun Z, Voigt T, Shah SP. Rheometric and ultrasonic investigations of viscoelastic properties of fresh Portland cement pastes. Cem Concr Res 2006;36(2):278-87.

[23] Betioli AM, Gleize PJP, Silva DA, John VM, Pileggi RG. Effect of HMEC on the consolidation of cement pastes: isothermal calorimetry versus oscillatory rheometry. Cem Concr Res 2009;39(5):440-5.

[24] Martins R, Bombard A. Rheology of fresh cement paste with superplasticizer and nanosilica admixtures studied by response surface methodology. Mater Struct 2012;45(6):905-21.

[25] Krieger IM, Dougherty TJ. A mechanism for non-Newtonian flow in suspensions of rigid spheres. Trans Soc Rheol 1959;3(1):137-52.

[26] Kaci A, Bouras R, Chaouche M, AndrÈani PA, Brossas H. Adhesive and rheological properties of mortar joints. Appl Rheol 2009;19(5).

[27] Lei W-G, Struble LJ. Microstructure and flow behavior of fresh cement paste. J Am Ceram Soc 1997;80(8):2021-8.

[28] Corr DJ, Juenger MCG, Monteiro PJM, Bastacky J. Investigating entrained air voids and Portland cement hydration with low-temperature scanning electron microscopy. Cem Concr Compos 2004;26(8):1007-12.

[29] Roussel N, Ovarlez G, Garrault S, Brumaud C. The origins of thixotropy of fresh cement pastes. Cem Concr Res 2012;42(1):148-57.

[30] Chang SH, Ryan MH, Gupta RK. The effect of $\mathrm{pH}$, ionic strength, and temperature on the rheology and stability of aqueous clay suspensions. Rheol Acta 1993;32(3):263-9. 